\title{
Exploration of Doped Semiconductors at the Atomic Scale
}

\author{
Alfonso Rodil ${ }^{1}$, Christian Krammel ${ }^{1}$, Rianne Plantenga ${ }^{1}$, Sebastian Kölling ${ }^{1}$, and Paul Koenraad ${ }^{1}$. \\ 1. Department of Applied Physics \& Institute for Photonic Integration, Eindhoven University of \\ Technology, Eindhoven, the Netherlands.
}

The controlled incorporation of doping atoms is essential for nearly all semiconductor devices. Devices such as transistors, light-emitting diodes, solar cells etc. are all impossible without the application of doping atoms that locally control the Fermi-level and the internal potential landscape. In such devices precise control over 3D distribution of the various doping species is thus critical for their functionality. The control over doping atoms in devices has steadily advanced in recent years to a level where on occasion the precise incorporation of just a single dopant atom is desired. For instance in the field of solotronics [1] one has moved beyond the use of doping atoms to locally control the Fermi level and one aims to base the functionality of the device on just one single doping atom that is placed at an exact position in the device. These factors force us to look for microscopic techniques that can visualize doping incorporation in semiconductor devices and materials at the atomic scale. In this presentation I will present some of our recent results with Scanning Tunneling Microscopy (STM) and Atom Probe Tomography (APT) in the study of doping atoms.

We have used cross-sectional STM to explore isovalent doping atoms in GaAs and InP and were able to observe isovalent doping atoms such as $\mathrm{Bi}$ in InP up to several monolayers below the cleaved surface, see Figure 1. Although the doping atoms do not introduce any free carries in the semiconductor material, due to a strain mismatch and a difference in the electron negativity a charge redistribution around the isovalent doping atoms will occur. We have been able to visualize this charge redistribution and to explain the strong anisotropy by Tight Binding Model simulations, see Figure 2.

Nanowires comprise a new class of highly interesting 3D semiconductor nanostructures in which doping also plays a crucial role. For their application in devices such as solar cells and light emitting diodes it is crucial to control the doping profile in them. Cross-sectional STM, which has an unprecedented capacity to determine the distribution and properties of doping atoms in epitaxially grown semiconductors is unfortunately not yet applicable for the study of doping atoms in Nanowires. The best and probably only technique that allows addressing this problem is Atom Probe Tomography. We have developed a new approach [2] in which we have been able to measure doping concentrations with Atom Probe Tomography down the ppm-level or a doping level slightly below $10^{17}$ atoms $/ \mathrm{cm}^{3}$. We have used this approach to examine $\mathrm{Si}$ (n-type) and $\mathrm{Zn}$ (p-type) doped InP nanowires and were able to extract the doping profiles and check dopant incorporation efficiency, see Figure 3a. We noticed that at high doping concentrations the growth of the nanowire could become unstable. Under these conditions a strong local variation of the doping concentration can occur resulting in dopant clusters, see Figure $3 b$. Finally we have used the Atom Probe Tomography technique to analyze details in the doping profiles that allow us to retrieve important details in the incorporation process for doping atoms in nanowires, see Figure 4.

\section{References:}

[1] P. M. Koenraad and M.E. Flatté, Nature Materials 10, (2011), 96.

[2] S. Koelling et al, "Atom-by-Atom Analysis of Semiconductor Nanowires with Parts Per Million Sensitivity” Nano Letters DOI: 10.1021/acs.nanolett.6b03109. 

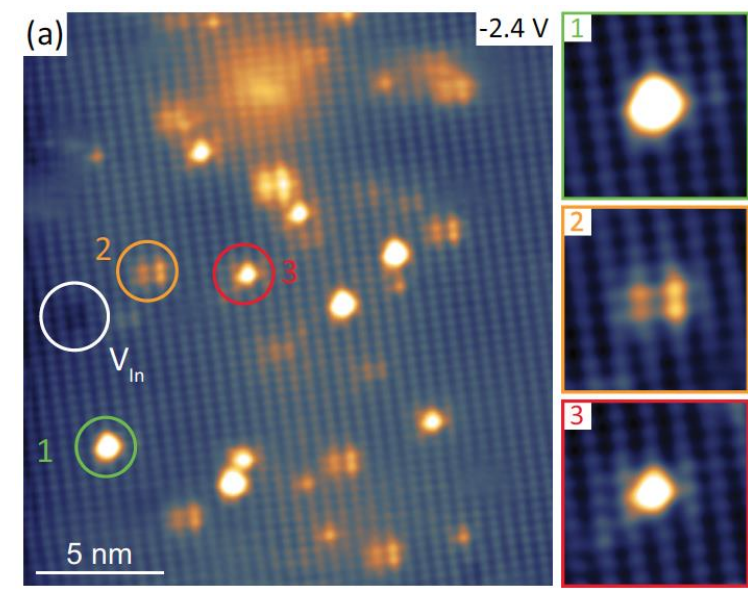

Figure 1. Cross-sectional STM image of a cleaved Bi doped InP sample. We can directly observe the spatial distribution of $\mathrm{Bi}$ isovalent doping atoms up to three monolayers below the cleavage surface.
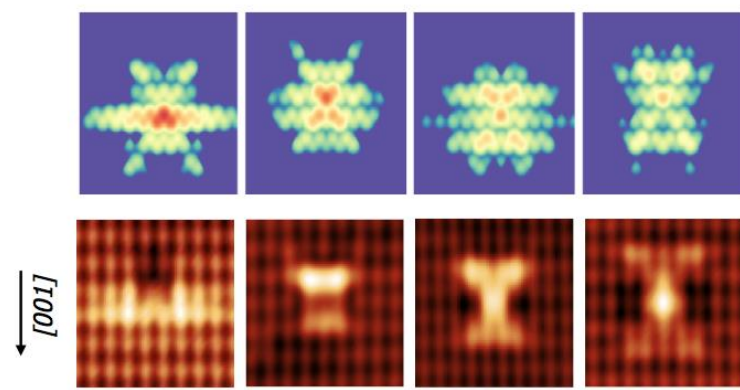

In top layer

$1 \mathrm{ML}$ below

2 ML below

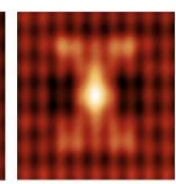

3 ML below

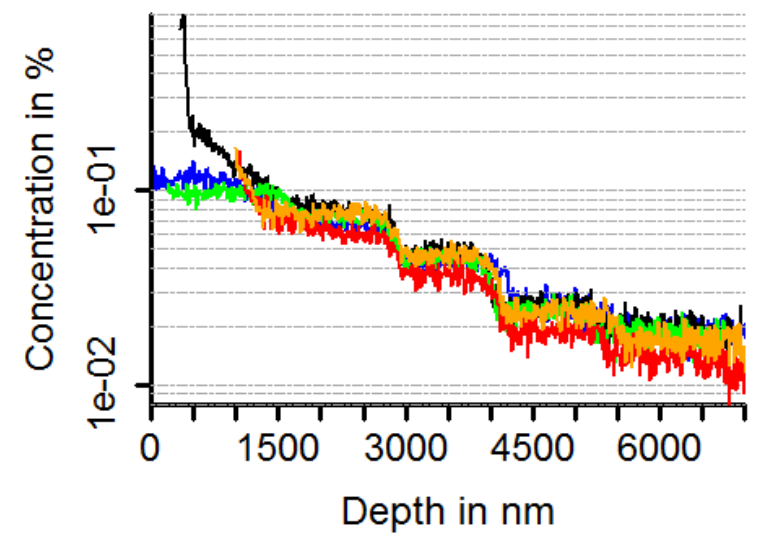

b)

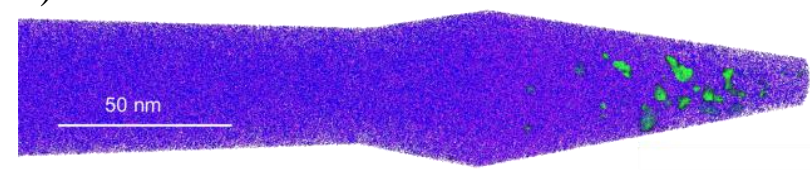

Figure 3. a) Si-doping profiles observed by Atom Probe Tomography in InP nanowires. b) Si doping clusters as indicated by the green iso-surfaces observed in a strongly doped InP nanowire.
Figure 2. Charge redistribution as measured around individual $\mathrm{N}$-atoms in in GaAs at different depths below the cleaved surface (lower panels). Calculated charge redistribution (upper panels) for the experimental cases shown in the lower panels.

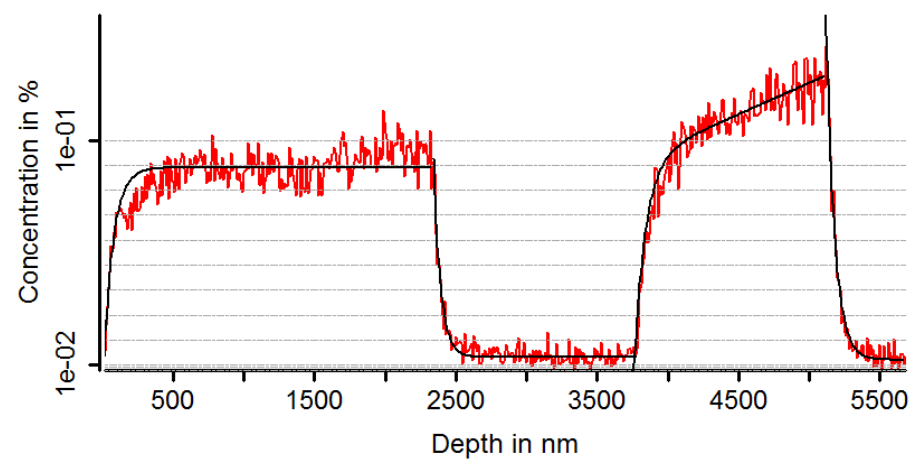

Figure 4. Si-doping profile in an InP nanowire as obtained by Atom Probe Tomography. Muraki-type fits where used to describe the quality of the doping interfaces. 